\title{
TEORÍAS PERSONALES DE DOCENTES DE LENGUA SOBRE LA ENSEÑANZA DE LA ESCRITURA EN SISTEMA DE EDUCACIÓN PÚBLICA CHILENA
}

\section{LANGUAGE TEACHER PERSONAL THEORIES ABOUT TEACHING WRITING IN THE CHILEAN PUBLIC EDUCATIONAL SYSTEM}

\author{
Roxanna Carolina Correa Pérez ${ }^{*}$ \\ Mónica Tapia Ladino \\ Angie Neira Martinez \\ Mabel Ortiz Navarrete
}

\section{RESUMEN}

Es sabido que las ideas que los docentes sostienen acerca de los procesos de enseñanza -aprendizaje de lenguas inciden en su quehacer pedagógico. Son escasos los estudios que explican las teorías personales de profesores de lenguas en torno a las habilidades lingüísticas de sus estudiantes. Por esta razón, el objetivo de este trabajo es determinar cuáles son las teorías personales sobre escritura y su enseñanza en la educación media de seis profesores de inglés y de seis de Lenguaje y Comunicación que se desempeñan en establecimientos educacionales municipalizados de dos regiones de Chile. Para acceder a las teorías personales, los docentes fueron entrevistados con la finalidad de conocer sus ideas sobre diferentes aspectos de la enseñanza de la escritura. La metodología de análisis fue cualitativa y el procesamiento de los datos se realizó con el software NVivo. Los resultados obtenidos indican que los docentes asignan una alta importancia al desarrollo de la escritura, realizan actividades secuenciadas, se apoyan en textos modelos, favorecen el trabajo individual y promueven la escritura de textos breves.

Palabras-clave: teorías personales; escritura; análisis cualitativo.

\section{ABSTRACT}

It is well known that the teachers' ideas about any area related to language teaching and learning processes impact on their pedagogical practice. There are not many studies, however, that explain language teachers' personal theories about linguistics abilities. The

* Universidad Católica de la Santísima Concepción.Chile, Chile. rcorrea@ucsc.cl; Universidad Católica de la Santísima Concepción.Chile, Chile. mtapia@ucsc.cl; Universidad de Concepción, Chile. angieneira@gmail.com; Universidad católica de la Santísima Concepción.Chile, Chile. mortiz@ucsc.cl 
aim of this article is to present the personal theories about writing of 6 English teachers and 6 Spanish teachers, who work in public schools in two regions of Chile. In order to find out the personal theories, we interviewed the teachers to deal with different aspects of the process of teaching writing. The methodology was qualitative and the data analysis was carried out with the support of NVivo software. The results indicate that the teachers assign great importance to the development of writing, carry out sequenced activities, support the process with model texts, foster individual work and promote the writing of brief texts. Keywords: personal theories; writing; qualitative analysis.

\section{ANTECEDENTES}

La experiencia de los docentes de lengua - ya sea materna o extranjera - con la enseñanza de la escritura puede resultar muy valiosa para implementar cambios educativos profundos en esta materia. Para conocer dicha experiencia, es necesario averiguar cómo los docentes comprenden los modos de enseñanza de la escritura, cómo evalúan los escritos de sus estudiantes, qué estrategias perciben beneficiosas en el proceso de enseñanza - aprendizaje, qué valor le asignan a la producción escrita y cómo abordan el proceso de enseñanza en el aula, entre otras interrogantes. Dado lo anterior, el objetivo de este artículo es presentar las teorías personales sobre escritura declaradas por un grupo de docentes de Enseñanza Secundaria de Español como Lengua Materna y de Inglés como Lengua Extranjera que se desempeñan en establecimientos educacionales municipales de dos regiones del sur de Chile.

\section{MARCO TEÓRICO}

\subsection{Los sistemas de creencias docentes}

El estudio de las experiencias de los docentes acerca de diferentes ámbitos de la enseñanza se ha centrado en el análisis de lo declarado y de lo observado sobre su actuación. Estas investigaciones tienen como objetivo develar las creencias o conceptos de los docentes acerca del proceso de enseñanza y aprendizaje. Específicamente, los estudios se han centrado en dilucidar aspectos como la actuación del docente en el aula, las metodologías implementadas, los sistemas de evaluación o el dominio de conocimiento disciplinar específico. El interés por conocer las ideas que subyacen a las prácticas pedagógicas surge del supuesto que señala que la actuación en el aula está guiada por las ideas o experiencias del docente. Desde esta perspectiva, cualquier innovación en el área de la enseñanza requiere, 
en primer lugar, conocer el pensamiento del profesorado, para luego, trabajar en los cambios que sean necesarios.

El desarrollo de la investigación en el área de la cognición docente ha sido abordado por diferentes autores y desde distintas perspectivas. Destacan los estudios de Spada (1992) quien propone denominar la experiencia docente declarada como conocimiento pedagógico específico (specific pedagogical knowledge). Con este término, el autor alude al dominio que un docente tiene sobre el conocimiento relacionado con un contenidoparticular. Por su parte, Golombek (1998) prefiere denominar a este tipo de experiencia como conocimiento práctico personal (personal practical knowledge - PPK) para lo que incorpora una perspectiva ética - afectiva al conocimiento del docente con respecto a situaciones educativas más allá del dominio conocimientos particulares. Woods (1996) opta por llamar a estas creencias como supuestos y conocimientos (beliefs, assumptions, knowledge BAK), pues estima que corresponden a constructos complejos que incluyen creencias y conocimientos de los docentes sobre los procesos de enseñanza de una lengua. Esta última propuesta amplía la idea de lo que se entiende por conocimiento del profesor, pues incorpora tanto los supuestos que el docente ha asimilado en su experiencia previa como aprendiz de una lengua como aquellos adquiridos en su práctica profesional.

Los distintos enfoques sobre las experiencias pedagógicas declaradas por los docentes se centraron en el área de la toma de decisiones y efectividad del profesor, es decir, en el desempeño observable tanto en su práctica docente como el análisis de los materiales de planificación. Estudios recientes se han focalizado en los procesos de comprensión del conocimiento del profesor, es decir, la cognición docente. En esta línea se encuentran los aportes de Fenstermacher (1994) y Orton (1996) quienes afirman que la separación de las nociones de creencias y conocimiento del profesor es problemática, pues la cognición docente es un concepto multidimensional que incluye las creencias, los conocimientos, los saberes relacionados con el contexto escolar, la práctica docente, el perfeccionamiento, entre otros. Para aprehender este fenómeno, Borg (2003) propone cognición docente como un término inclusivo que cubre la complejidad de la vida mental de los profesores, es decir, lo que estos piensan, saben o creen en relación con el ejercicio de su trabajo.

En el ámbito chileno, Díaz y Solar (2011) proponen el concepto de creencias y lo entienden como "aquellos constructos mentales eclécticos que provienen de diferentes fuentes: experiencias personales, prejuicios, juicios, ideas, intenciones" (p.109). Desde esta perspectiva, los autores conciben la realidad docente como un fenómeno subjetivo relacionado con su actuación. De este modo, los constructos de pensamiento que elaboran los docentes presentan características que los definen. 
Por ejemplo, ellas no son directamente observables, pues se elaboran a partir de experiencias cotidianas en contextos de interacción comunicativa y social, y que actúan como filtros al interpretar información y experiencias nuevas (BAILEY, CURTIS y NUNAN, 2001; PHIPPS y BORG, 2007). Por lo tanto, se trata de sistemas subjetivos que se elaboran paulatina y progresivamente durante toda la vida del docente.

Una vez delimitada el área que cubren los estudios de la cognición docente, es necesario describir los rasgos que caracterizan a estos fenómenos. Diversos autores coinciden en que las creencias que se establecen tempranamente en la vida de los profesores son resistentes al cambio, incluso en un contexto de contradicciones evidentes. Al respecto, Pajares (1992) señala que la resistencia tiene relación con la dimensión emocional del docente. Sin embargo, Borg (1999) señala que los profesores pueden replantear sus teorías si el contexto o temática instruccional es incierta o no está claramente definida. En este caso, entran en tensión las creencias del profesor sobre aspectos de enseñanza específica con las demandas pedagógicas de la institución o el contexto en el que se desempeña. Almarza (1996) propone una explicación a dicho fenómeno al plantear que existe una diferencia entre los cambios cognitivos y los cambios conductuales de los docentes. Para el autor, las modificaciones conductuales son susceptibles de ser alteradas de manera más eficaz a través de programas de capacitación o perfeccionamiento. Cabaroglu y Roberts (2000), por su parte, identifican los posibles cambios que se pueden provocar en el desempeño docente, para lo cual se preguntan sobre

¿Cuál es la naturaleza de los cambios observados en la estructura (lo que el profesor piensa) y contenido (la manera en que los individuos jerarquizan y organizan sus sistema de creencias) de las teorías personales de los profesores, en diferentes etapas de su programa de formación? (p.234).

La respuesta que ofrecen los autores indica que, a pesar de que no es posible lograr modificaciones significativas en los contenidos de las teorías personales, sí es posible variar su organización y jerarquización. Es decir, es factible influir en la ordenación o sistematización de los contenidos de las teorías personales o creencias de los profesores, por lo tanto, parecen viables los programas de perfeccionamiento orientados a provocar cambios en el desempeño docente.

Por su partea, Peng (2011) señala que, en el ámbito de la enseñanza de una segunda lengua, las creencias del docente son susceptibles al cambio. Tales variaciones, que pueden ocurrir en diferentes momentos del desempeño profesional, son influenciadas por aspectos micro y macrocontextuales como las emociones y la 
autopercepción de la enseñanza. Dada la multidimensionalidad del fenómeno, Peng (2011) afirma que la trayectoria o evolución de las teorías personales es compleja.

Para efectos de esta investigación, adoptamos la propuesta de Sendan y Roberts (1998) sobre teorías personales quienes las entienden como un sistema subyacente de constructos a los que los profesores en formación y en ejercicio recurren cuando evalúan, clasifican, guían y piensan sobre su práctica pedagógica. Al mismo tiempo, entendemos que dichos constructos se pueden modificar, ya sea en su reorganización interna o en su fluctuación temporal evidenciada en el desempeño en el aula.

\subsection{Teorías personales sobre la enseñanza de la escritura}

La investigación sobre la cognición docente centrada en la enseñanza de la lengua - materna o extranjera - ha dado cuenta de las teorías personales de los docentes sobre procesos de enseñanza de escritura. Estos estudios han abordado diversos aspectos asociados con la producción escrita, como la gramática, los enfoques de enseñanza de la escritura o las metodologías implementadas en el aula.

En el área de la enseñanza de la gramática, Borg (1999) señala que los docentes tienden a aplicar diferentes metodologías como los métodos descriptivos, inductivos o el aprendizaje por descubrimiento. Sin embargo, los resultados revelan que, en general, los docentes no son conscientes de las implicancias de la aplicación de una metodología en los estudiantes o en su labor docente. En esta línea Tsui (1996) señala que se producen tensiones entre los factores institucionales y la capacidad del docente para implementar metodologías que considera adecuadas para el desarrollo de la escritura. El autor revela que, en ocasiones, las condiciones institucionales limitan las decisiones metodológicas que los profesores consideran necesarias para la enseñanza de la escritura.

Cuando se han estudiado las teorías personales sobre los enfoques de enseñanza de la escritura, diversos autores (GRAHAM, HARRIS, MACARTHUR y FINK, 2002; HILLOCKS, 1984; LIPSON, MOSENTHAL, DANIELS y WOODSIDE-JIRON, 2000) coinciden en identificar los aspectos instruccionales más señalados por los docentes, a saber, la selección del tópico, las limitaciones del tiempo de escritura, la enseñanza de las habilidades necesarias para escribir de manera aislada y los aspectos mecánicos de la escritura. En el caso específico de la enseñanza de la escritura en una segunda lengua, Eisenstein - Ebsworth y Schweers (1997) plantean que la experiencia de los docentes como aprendices de una segunda lengua tiene una influencia significativa en su práctica docente. Es 
decir, la experiencia previa del docente sobre cómo él mismo aprendió la lengua se relaciona con ciertas creencias cognitivas que forman la base de una conceptualización inicial que determina el modo como enfrentan la enseñanza de una segunda lengua. Por lo tanto, tal estimación puede influenciar su práctica docente. En este mismo sentido, Woods (2011) señala que la personalización de las teorías se hace evidente en la elección de las habilidades comunicativas que el docente escoge enfatizar a la hora de enseñar lengua. La personalización explicaría por qué en ocasiones las elecciones metodológicas de los docentes no se relacionan del todo con las teorías de enseñanza de lengua. Más bien se basan, según el autor, en las experiencias personales vivenciadas durante su propio proceso de formación.

Algunos autores como Nunan (1992) y Gatbonton (1999) han investigado las razones declaradas por los docentes para explicar sus decisiones metodológicas o de enseñanza. Según Gatbonton (1999) las teorías personales de los profesores se focalizan en aspectos del manejo del lenguaje como explicación de vocabulario y la creación de contextos significativos para desarrollar la escritura. Los resultados de la investigación de Nunan (1992), por su parte, revelan que los docentes priorizan aspectos como el manejo de la lengua, el ritmo y duración de la clase, el tiempo de habla del profesor y la calidad de sus explicaciones e instrucciones. En esta línea, Burns (1992) describe cinco aspectos que parecieran influir en la enseñanza y en los enfoques adoptados por los docentes, esto es, la naturaleza del lenguaje y su relación con el aprendizaje inicial, la relación entre lenguaje hablado y escrito, la naturaleza del aprendizaje inicial de una lengua y las estrategias relevantes para su aprendizaje, las características de los estudiantes en términos de su habilidad para aprender, y la naturaleza del lenguaje de la clase y el rol del docente en ella. Los elementos identificados por Borg (1999) revelan que existen diferentes variables que determinan el proceso de enseñanza-aprendizaje de una lengua.

En un sentido diferente, pero complementario, Tsui (1996) investiga sobre los principales dilemas que el profesor enfrenta cuando aplica la metodología de escritura como proceso. Los principales desafíos que identifica el autor se relacionan con la ansiedad y frustración que provoca en el docente tanto la implementación de la metodología de enseñanza de la escritura como el periodo de perfeccionamiento necesario para adoptarla.

Finalmente, los hallazgos de Feryok (2010) relevan el rol de la reflexión en los procesos de reorganización de las teorías personales. Al respecto, señala que si la práctica docente en el aula produce disonancia o tensión cognitiva en el profesor, esto contribuiría a focalizar su atención en dicho elemento. Esta atención llevaría a 
la reflexión que promueve el cambio cognitivo necesario para la adopción de nuevas prácticas.

En términos generales, es posible señalar que las teorías personales de los docentes sobre la enseñanza de un aspecto específico de la lengua, en este caso, los procesos de enseñanza-aprendizaje de la escritura en lengua materna y extranjera, orientan las decisiones metodológicas de los docentes, ya sea excluyendo algún aspecto o adicionando técnicas de enseñanza. La revisión bibliográfica revela que las experiencias personales relacionadas con el aprendizaje de la lengua persisten en la práctica del docente. Lo más relevante, a nuestro juicio, es que las teorías personales pueden ser reorganizadas y modificadas por el profesor cuando media un proceso de perfeccionamiento o si se promueve la reflexión metacognitiva acerca de su desempeño profesional. Desde esta perspectiva, la presente investigación aborda el estudio de las teorías personales acerca de la escritura que expresan los profesores de Inglés y, de Lenguaje y Comunicación de Enseñanza Media del sistema educacional público chileno, con el objetivo de identificar cómo son los constructos de pensamiento que se observan en ellos.

\section{DISEÑO DE LA INVESTIGACIÓN}

Este estudio se enmarca en el paradigma de la investigación acción, dado que fue realizado por docentes investigadores interesados en averiguar sobre el rol de la escritura en el proceso de enseñanza-aprendizaje (MILLS, 2007; MERTLER, 2006). La investigación acción aborda una indagación sistemática conducida por profesores para conseguir información sobre cómo operan los estudiantes, cómo aprenden y cómo enseñan los docentes (MERTLER, 2006, p. 2).

La metodología de investigación es cualitativa, pues pretende explicar razones del comportamiento de los sujetos participantes analizando su discurso sobre el proceso de enseñanza - aprendizaje de la escritura, tanto en lengua materna (español) como en lengua extranjera (inglés). Dada la naturaleza cualitativa del análisis, se trata de un estudio de caso, pues se pretende estudiar un fenómeno a partir del estudio en profundidad de variadas fuentes (BISQUERRA, 2009). Específicamente, se analizan cualitativamente los discursos de un grupo de docentes para identificar y caracterizar sus teorías personales sobre la enseñanza de la escritura. 


\subsection{Participantes}

Los participantes de este estudio fueron doce profesores de enseñanza secundaria, seis del subsector Lenguaje y Comunicación y seis del subsector Inglés. Dichos docentes pertenecen a establecimientos públicos de las Regiones del Bío Bío y de la Araucanía del sur de Chile. La obtención se realizó mediante la aplicación de un criterio intencionado, pues se trata del conjunto de docentes que se interesó en participar de manera voluntaria en una capacitación de 120 horas pedagógicas sobre la incorporación de las wikis al aula para favorecer el aprendizaje de la escritura colaborativa.

El rango de años de servicio de los docentes participantes va de 1 a 26 años. En cuanto a la distribución geográfica de los establecimientos en los que se desempeñan, nueve de ellos ejercen su profesión en la región del Bío Bío, particularmente en las comunas de Curanilahue, Cobquecura, Coronel y Hualpén, y los tres restantes en la Región de la Araucanía, específicamente, en la comuna de Purén.

\subsection{Recolección de datos}

La tarea de recolección de los datos se realizó durante la primera fase de una investigación mayor. Los datos que presentamos en este trabajo fueron obtenidos a partir de entrevistas semiestructuradas realizadas a los docentes al comienzo de un periodo de capacitación orientado a implementar en el aula secuencias didácticas para desarrollar habilidades de escritura en ambientes on line que favorecen la producción de textos escritos colaborativos.

La entrevista semiestructurada, que fue diseñada por las investigadoras, abordó temas como: ideas y experiencias con escritura, importancia, metodología, proceso y evaluación de la escritura, tipo de escritos enseñados, dificultades y recursos de apoyo. Las preguntas de las entrevistas fueron piloteadas y validadas previamente mediante su aplicación a docentes que no participaron en esta investigación. Para la realización de las entrevistas, se utilizó un protocolo que favoreció que la actividad se realizara de manera similar; además, se contó con el consentimiento informado de los participantes. Todas las entrevistas se realizaron en salones de clases de la Universidad Católica de la Santísima Concepción y fueron recogidas con ayuda de grabadoras digitales. Los materiales fueron almacenados en archivos de audio, los que fueron utilizados para la realización de transcripciones a ortografía corriente. 


\section{ANÁLISIS DE DATOS}

Una vez transcritas las entrevistas en procesador Word, fueron almacenadas y archivadas con un código identificatorio. El análisis de la información se realizó siguiendo el esquema de tareas para el análisis de datos propuesto por Miles y Huberman (1994), quienes plantean que en el procesamiento de los datos cualitativos se distinguen tres fases: la primera, denominada etapa de reducción de los datos; la segunda, la etapa de síntesis y agrupamiento y, finalmente, la etapa de verificación de los resultados. Las codificaciones se realizaron con apoyo del software QSRNVivo, que facilitó la tarea de almacenamiento, sistematización y codificación de los textos. Dicho software permite organizar y analizar información proveniente de diferentes fuentes. Se trata de una herramienta que facilita el proceso de construcción de teorías a partir de la codificación y el manejo de gran cantidad de información en forma de textos digitalizados.

Para la etapa de reducción de los datos, se procedió a leer detenidamente las entrevistas y a aislar los segmentos de los discursos de los docentes según los temas previamente definidos y los temas emergentes que surgieron de las entrevistas. Cada segmento fue asociando a temas y subtemas, labor facilitada por el sistema de organización, almacenamiento y clasificación que ofrece el programa QSRNvivo.

Para efectos de este artículo, daremos cuenta del análisis de las siguientes categorías: (1) ideas sobre escritura, (2) habilidades necesarias para escribir, (3) importancia de la escritura, (4) frecuencia de la escritura en el aula, (5) metodología y estrategias de enseñanza de la escritura, (6) organización de los estudiantes para las tareas de escritura y (7) proceso y actividades de escritura realizadas. Seleccionamos tales nodos dado que presentaron una rica densidad de temas y subtemas asociados.

\section{RESULTADOS}

Comentamos los resultados en función de las categorías mencionadas previamente y a las subcategorías identificadas en el proceso de análisis de los datos. Para ello, ofrecemos extractos de los discursos de los docentes entrevistados que representan las ideas que más se asocian a las categorías identificadas.

En primer término, revisamos las ideas sobre la enseñanza de la escritura que expresan los docentes de Inglés y, de Lenguaje y Comunicación. Presentamos los siguientes extractos: 
Tabla 1. Ideas de los docentes sobre la enseñanza de la escritura

\begin{tabular}{|l|l|}
\hline \multicolumn{1}{|c|}{ Subsector } & \multicolumn{1}{|c|}{ Fragmento } \\
\hline Inglés1 & $\begin{array}{l}\text { "Muy difícil, especialmente en inglés... porque hacer ensayos o cartas es muy } \\
\text { estructurado donde tienes que seguir muchas reglas y manejar mucho vocabulario } \\
\text { avanzado para poder escribir en inglés, ...." }\end{array}$ \\
\hline L y C 7 & $\begin{array}{l}\text { "Yo creo que es complicado desde el punto de vista de la formalidad, porque } \\
\text { si bien vivimos reproduciendo textos orales, discurso, llevarlo a la escritura al } \\
\text { aspecto más formal, es más bien complicado." }\end{array}$ \\
\hline
\end{tabular}

Las dificultades identificadas por los profesores de Lenguaje y Comunicación revelan que la tarea de enseñar a escribir demanda un doble esfuerzo: enseñar la habilidad de escribir y favorecer el uso de la lengua formal. De este modo, los docentes asocian escritura al dominio de una variedad de la lengua, lo que exige más tiempo de enseñanza que el abordaje de otras temáticas del currículo. Cuando los docentes se refieren a las habilidades necesarias para escribir, coinciden en señalar que escribir es una habilidad que requiere conocimientos de distinto orden, por ejemplo, conocimientos sobre el tipo de texto, conocimientos sobre los rasgos que permitan expresar la postura crítica del escritor sobre el tema, entre otros. El análisis de las entrevistas revela que tanto los docentes de Lenguaje y Comunicación como los de Inglés consideran que la producción escrita es un proceso complejo. Para ellos, enseñar a escribir es una tarea que requiere considerar desde los aspectos metodológicos hasta los contextuales, como el tiempo de clases, la motivación de los estudiantes, los recursos disponibles, entre otros. Sin embargo, advertimos que existe una ligera diferencia en los aspectos declarados como complejos: mientras los docentes de Inglés identifican entre las dificultades la falta de ideas y vocabulario, los de Lenguaje y Comunicación ( $\mathrm{LyC}$ ) enfatizan en los aspectos motivacionales relacionados con el acto de escribir. A continuación, se presentan algunos extractos de lo declarado por los docentes:

Tabla 2. Habilidades necesarias para desarrollar la escritura

\begin{tabular}{|l|l|}
\hline Sujeto & Fragmento \\
\hline Inglés 4 & $\begin{array}{l}\text { "Yo creo que tiene que tener, en primer lugar, el vocabulario y después la habilidad } \\
\text { para expresar las ideas, expresar la opinión, poder pasar lo que uno piensa al papel, } \\
\text { escribir bien. Igual es importante, ordenar las ideas, secuenciarlas ... que esté bien } \\
\text { organizado el texto..." }\end{array}$ \\
\hline Ly C 3 & $\begin{array}{l}\text { "Habilidades de redacción, habilidades básicas como comunicativas de expresar las } \\
\text { ideas, coherencia y cohesión para que lo exprese por escrito se entienda y no se mal } \\
\text { interprete." }\end{array}$ \\
\hline
\end{tabular}




\begin{tabular}{|l|l|}
\hline Ly C 5 & $\begin{array}{l}\text { "Reflexión, sintetizar, comprender y tener una postura bastante crítica, dependiendo } \\
\text { del contexto en que se esté hablando. Son habilidades que son necesarias. } \\
\text { Principalmente sintetizar que es una habilidad sumamente compleja." }\end{array}$ \\
\hline
\end{tabular}

Los docentes de ambos subsectores coinciden en que para escribir es necesario tener conocimiento sobre el tema que se quiere abordar y habilidad para expresar las ideas. En particular, los docentes de LyC precisan más sobre aspectos discursivos como la organización de las ideas, la coherencia y cohesión. En cambio, los docentes de Inglés insisten en el manejo de vocabulario como punto de partida y, posteriormente, la organización del texto. El análisis de esta categoría revela que los docentes convergen en lo que Gatbonton (1999) y Nunan (1992) denominan el manejo del lenguaje, es decir, aquellos aspectos estructurales que otorgan cohesión y coherencia a un texto escrito. Sin embargo, se aprecia que para los docentes de Inglés la enseñanza de vocabulario constituiría la piedra angular del desarrollo de la escritura, antes que la organización de las ideas. Desde esta perspectiva, reconocen la importancia del componente léxico, priorizándolo por sobre otros aspectos de la construcción de un texto.

En tercer término, acerca de la importancia de la escritura, los docentes dan cuenta de cómo aprender a escribir textos de diversa índole contribuye al desarrollo de los estudiantes. A continuación se presentan algunas de las respuestas de los profesores:

Tabla 3. Contribución de la escritura al desarrollo de los estudiantes

\begin{tabular}{|l|l|}
\hline \multicolumn{1}{|c|}{ Sujeto } & \multicolumn{1}{c|}{ Fragmento } \\
\hline Inglés 2 & $\begin{array}{l}\text { "Le daría mucho más herramienta a su desarrollo (de los estudiantes). Se les } \\
\text { abriría un mundo de oportunidades que ellos no vislumbran" }\end{array}$ \\
\hline LyC 6 & $\begin{array}{l}\text { "Potencia todas sus habilidades cognitivas. Si el alumno no sabe escribir es, } \\
\text { porque no sabe hablar y no sabe pensar" }\end{array}$ \\
\hline LyC 5 & $\begin{array}{l}\text { "Bastante lo que es el aspecto cognitivo más que nada, o sea, tratar de } \\
\text { sintetizar, ordenar, en primer lugar, todas las ideas que tengan, aporta } \\
\text { bastante" }\end{array}$ \\
\hline
\end{tabular}

Los datos analizados evidencian que los profesores participantes en esta investigación valoran diferentes aspectos del rol de la escritura en el desarrollo de los estudiantes. Por una parte, los docentes de Inglés se centran principalmente en las oportunidades futuras que ofrece el aprendizaje de una lengua extranjera, por otra, los docentes de Lenguaje y Comunicación se centran en los aspectos cognitivos que se desarrollan gracias a la escritura, por lo tanto, valoran tal aprendizaje como 
una herramienta que favorece el desarrollo del pensamiento. Las divergencias en las teorías personales de los profesores de Inglés y, de Lenguaje y Comunicación en relación con la importancia de la escritura demuestran que existe una visión y un propósito distinto en la enseñanza de una lengua materna que en la de una lengua extranjera.

En cuarto término, acerca de la frecuencia de escritura en el aula, los docentes señalan lo siguiente:

Tabla 4. Frecuencia de actividades de escritura en el aula

\begin{tabular}{|l|l|}
\hline Sujeto & \multicolumn{1}{c|}{ Fragmento } \\
\hline Inglés 2 & $\begin{array}{l}\text { "La verdad que muy poca, el tema es que a lo que tú me dices escritura, en realidad, son } \\
\text { actividades como completar párrafos. Los estudiantes no saben escribir adecuadamente } \\
\text { en español, por lo tanto, en inglés es más difícil aún". }\end{array}$ \\
\hline Inglés 3 & $\begin{array}{l}\text { "Casi siempre lo que más trabajo es escritura, lectura. No trabajo mucho lo que es } \\
\text { habilidad auditiva en inglés. Me gusta darles siempre tareas de lectura, la comprensión } \\
\text { lectora es muy importante se evalúa en todas partes SIMCE, PSU en todo esto es } \\
\text { comprensión lectora, nada más". }\end{array}$ \\
\hline LyC3 & $\begin{array}{l}\text { "La mayoría de las clases, aunque sea un texto breve, pero que escriba" } \\
\text { "La realidad donde estoy inserta vienen con falencias muy graves, entonces hay que } \\
\text { hacerlos escribir ir puliendo es un trabajo bastante complicado, porque es casi enseñarle } \\
\text { escribir de nuevo. Entonces si tiene que ser una actividad constante y prácticamente la } \\
\text { mayoría de las clases los hago escribir..." }\end{array}$ \\
\hline
\end{tabular}

Como se aprecia en los extractos, los docentes de Inglés declaran que normalmente no desarrollan tareas de escritura, pues no las consideran entre las habilidades prioritarias de la enseñanza del idioma. Las razones que subyacen a esta decisión son la falta de tiempo y las dificultades en el desempeño de la lengua materna de los estudiantes. Asimismo, señalan que los programas del MINEDUC (Ministerio de Educación de Chile) enfatizan el desarrollo de las habilidades de comprensión lectora y auditiva. Por su parte, los profesores de Lenguaje y Comunicación realizan tareas de escritura de manera frecuente, independientemente de los obstáculos, como tiempo de aula o falencias lingüísticas de los estudiantes. Los profesores de Lenguaje reconocen que enseñar a escribir es una tarea compleja, pero necesaria, por lo tanto, para garantizar el trabajo sistemático, optan por solicitar textos breves y de ese modo, se ajustan a las directrices del currículum nacional.

En quinto término, respecto de la metodología y estrategias de enseñanza de la escritura, se identificaron dos subcategorías: estrategias utilizadas y organización del aula. Algunas de las respuestas de los participantes son las siguientes: 
Tabla 5. Estrategias y organización del aula

\begin{tabular}{|l|l|}
\hline \multicolumn{1}{|c|}{ Sujeto } & \multicolumn{1}{c|}{ Fragmento } \\
\hline Inglés 2 & $\begin{array}{l}\text { "Les muestro ejemplos, jugamos un poco con la oraciones para internalizar un poco } \\
\text { el proceso. Los chicos observan las frases que se dicen, cómo se tiene que decir, } \\
\text { para luego ellos anotar lo que significa la expresión, por ejemplo. Entonces van } \\
\text { adquiriendo vocabulario y hacen lista de vocabulario". }\end{array}$ \\
\hline Inglés 3 & $\begin{array}{l}\text { "Con el famoso PPP, en el presentation que es la primera etapa, le presento los } \\
\text { contenidos, sobre todo si es gramatical: tiempo verbal, algún uso de algo en } \\
\text { específico, se los explico de manera oral. Después en el practice, muestro ejemplos } \\
\text { de ejercicios ocupando lo que se está enseñando. Y la última etapa, el production es } \\
\text { donde ellos emplean todo lo que hemos visto en el presentation y en el practice, pero } \\
\text { con un tópico especial". }\end{array}$ \\
\hline Ly C1 & $\begin{array}{l}\text { "Ellos deberían ya saber escribir en primero, segundo, tercero medio. Entonces yo } \\
\text { solamente les enseño a escribir los tipos de textos o, por ejemplo, poemas, prosas } \\
\text { que esas cosas que son particulares de cada unidad". }\end{array}$ \\
\hline L yC 6 & $\begin{array}{l}\text { "Por ejemplo, en Cuarto Medio con el discurso público, primero les presento } \\
\text { algunos discursos y los hago escuchar discursos. Luego los hago leer discursos, los } \\
\text { analizamos y, posteriormente, los hago que ellos hagan su propio discurso". }\end{array}$ \\
\hline
\end{tabular}

La revisión de los discursos seleccionados evidencia convergencias entre los docentes de Inglés y de Lenguaje y Comunicación. En cuanto a las estrategias utilizadas en el aula, ambos grupos utilizan la estrategia de "modelamiento" para enseñar a escribir, es decir, muestran otros textos como referencia para guiar la escritura. En el caso de los profesores de Inglés, proponen ejemplos de oraciones que quieren que los alumnos produzcan para llegar a construir un texto. Por su parte, los docentes de Lenguaje y Comunicación inician el proceso de escritura identificando el tipo de texto y analizando su organización discursiva. Llama la atención que uno de los docentes señale que no enseña a escribir, ello pudiera significar que asume la escritura como una actividad motora en la que se involucra la construcción oracional. Por lo tanto, invierte sus esfuerzos en los aspectos semánticos, discusivos y genéricos de los escritos. En cuanto a la organización de las actividades, lo declarado por los profesores indica que organizan sus actividades de enseñanza secuencialmente, lo que quiere decir que identifican momentos de aprendizaje y ordenan las tareas de manera temporal.

En sexto término, acerca de la organización de los estudiantes para las tareas de escritura, los docentes señalan lo siguiente: 
Tabla 6. Organización de los estudiantes en el aula

\begin{tabular}{|l|l|}
\hline \multicolumn{1}{|c|}{ Sujeto } & \multicolumn{1}{c|}{ Fragmento } \\
\hline Inglés 4 & $\begin{array}{l}\text { "A mí me gusta más el trabajo individual. No me gusta mucho el trabajo en } \\
\text { equipo, porque no falta que dos o tres trabajen y el resto no. También fui } \\
\text { estudiante y sé que eso pasa, me tocaba hacer a mí los trabajos y el resto no } \\
\text { los hacía, así que de repente los hago trabajar en parejas". }\end{array}$ \\
\hline Ly C 6 & $\begin{array}{l}\text { "En forma individual en la sala de clases. Generalmente cuando trabajan en } \\
\text { grupo siempre hay uno o dos que no trabajan y no hacen nada, distraen al } \\
\text { resto y reclaman después por las evaluaciones grupales". }\end{array}$ \\
\hline
\end{tabular}

Los profesores coinciden en que las tareas de escritura se realizan de manera individual o en pares, principalmente, debido a que los docentes consideran que la habilidad de escribir es una capacidad individual, por lo tanto, no es posible desarrollarla de manera grupal. Solo un docente de Lenguaje y Comunicación declaró que ocasionalmente realiza trabajo colaborativo. Por lo tanto, la tendencia general es la de promover la escritura individual y, en casos excepcionales, el trabajo grupal.

Es llamativo que los docentes desconfíen del modo cómo los estudiantes distribuyen las responsabilidades en el trabajo grupal. La experiencia personal y profesional les indica que en los grupos siempre se dan desequilibrios que obligan a unos a trabajar más y a otros, menos. Tal desconfianza revela la necesidad de conocer más acerca del trabajo colaborativo y de la asignación de responsabilidades debidamente descritas y asignadas.

Finalmente, en relación con el proceso y actividades de escritura realizadas, se desprenden dos subcategorías: los pasos o secuencias para enseñar a escribir y, la cantidad y organización del tiempo destinado a este proceso. Al respecto, los docentes declararon lo siguiente:

Tabla 7. Proceso de enseñanza de la escritura

\begin{tabular}{|l|l|}
\hline \multicolumn{1}{|c|}{ Sujeto } & \multicolumn{1}{|c|}{ Fragmento } \\
\hline Inglés 5 & $\begin{array}{l}\text { "Les enseño las partes de la oración: sujeto, verbo y objeto. Les enseño por parte } \\
\text { sobre el complemento y de varias formas la estructura de la oración. Primero } \\
\text { hablando, repitiendo. La mera repetición para los alumnos que quieren aprender } \\
\text { bien, memorizando o escuchando para ellos es más fácil después hacer un párrafo". }\end{array}$ \\
\hline Inglés 1 & $\begin{array}{l}\text { "Generalmente empiezo desde el vocabulario y hacemos oraciones. Después } \\
\text { pequeños textos con ejemplos míos. La biografía, mi biografía primero después } \\
\text { ellos van viendo y van cambiando la información. Después tiene que escribir de otra } \\
\text { persona y, generalmente, voy desde lo más pequeño hasta lo más avanzado". }\end{array}$ \\
\hline
\end{tabular}




\begin{tabular}{|l|l|}
\hline L y C 6 & $\begin{array}{l}\text { "Hacen un borrador en clases, a la segunda clase lo revisamos. En la tercera clase, lo } \\
\text { mejora y la cuarta clase queda el producto final". }\end{array}$ \\
\hline L y C 1 & $\begin{array}{l}\text { "Bueno, las dos horas para introducir el tema la estructura de un informe, por ejemplo. } \\
\text { Las semanas siguientes que ellos vayan desarrollando dentro de la clase ese informe } \\
\text { hasta que llegue un producto final". }\end{array}$ \\
\hline
\end{tabular}

En cuanto a los pasos o secuencias de actividades, se advierten diferencias sustanciales entre los profesores entrevistados. Los profesores de Inglés realizan actividades de escritura guiada, centradas predominantemente en las partes de la oración, es decir, se dedican a la microredacción. En cambio, los profesores de Lenguaje y Comunicación abordan el escrito de una manera más global y con un enfoque de enseñanza como proceso. Para ello, estos últimos dedican varias clases a la generación de un escrito, siguiendo las fases descritas por Flower y Hayes (1981) de planificación, textualización y revisión de los textos. Como los estudiantes tienen un conocimiento del sistema como lengua materna, los docentes asumen la enseñanza de la escritura como un proceso recursivo, en el que es necesario revisar constantemente las fases de elaboración de un escrito. En cambio, los docentes de Inglés prefieren asumir un enfoque bottom -up (FLESCH, 1955), es decir, de abajo hacia arriba, desde la oración al texto para apoyar a sus estudiantes en la construcción de los escritos.

Sobre la segunda subcategoría relacionada con el tiempo que dedican los profesores al aprendizaje de la escritura en el aula, presentamos los siguientes fragmentos:

Tabla 8. Tiempo dedicado al aprendizaje de la escritura

\begin{tabular}{|l|l|}
\hline \multicolumn{1}{|c|}{ Sujeto } & \multicolumn{1}{c|}{ Fragmento } \\
\hline Ing 4 & $\begin{array}{l}\text { "Casi nunca. Escriben al principio lo que yo escribo en la pizarra. Bueno eso es harto. } \\
\text { Pero generalmente que ellos escriban algo, no, que ellos redacten algo, no. Solo a } \\
\text { principio de año o en alguna actividad". }\end{array}$ \\
\hline Ing 5 & "No es tan frecuente. En las pruebas los hago escribir. Pero así como ejercicio, no." \\
\hline LyC 3 & $\begin{array}{l}\text { "La realidad donde estoy inserta vienen con falencias muy graves, entonces hay } \\
\text { que hacerlos escribir ir puliendo es un trabajo bastante complicado, porque es } \\
\text { casi enseñarle escribir de nuevo entonces tiene que ser una actividad constante y } \\
\text { prácticamente la mayoría de las clases los hago escribir". }\end{array}$ \\
\hline LyC 5 & $\begin{array}{l}\text { "Frecuentemente. Es un trabajo que hago en mi clase sobre todo con los alumnos de } \\
\text { primer año que escriben textos como cartas, informes. En Tercero Medio con el texto } \\
\text { argumentativo, escribir comentarios y opiniones, y sobre todo en las evaluaciones de } \\
\text { lectura. Ahí escriben, son todas preguntas de desarrollo". }\end{array}$ \\
\hline
\end{tabular}


Los datos revelan divergencias en el tema de la frecuencia con que los docentes de Inglés y, de Lenguaje y Comunicación abordan la escritura en el aula. Los profesores de lengua extranjera expresan que en muy pocas ocasiones realizan actividades tendientes a desarrollar la habilidad. Tales actividades se circunscriben básicamente a responder preguntas con oraciones breves. En cambio, los docentes de Lenguaje y Comunicación dedican más tiempo a la enseñanza de la escritura. De hecho, la frecuencia con que realizan actividades es una manera que les permite mejorar las falencias que presentan los estudiantes. La constante ejercitación y la evaluación mediante pruebas de desarrollo son estrategias que parecen generar resultados cuando ellas se repiten en el tiempo.

\section{DISCUSIÓN}

Los hallazgos recabados en esta investigación revelan que las teorías personales de los docentes entrevistados sobre los procesos de enseñanza - aprendizaje de la escritura evidencian similitudes y diferencias entre quienes enseñan Lenguaje y Comunicación o Inglés. Sobre la importancia que asignan a la habilidad de saber escribir, los docentes de Inglés expresan la idea de que el idioma permite ampliar las posibilidades laborales futuras de los estudiantes. Como señalan Castro, Mèndez y Sercu $(2003$, p. 1) "para el profesor de lenguas extranjeras, enseñar significa conectar a sus estudiantes con nuevas realidades culturales". Por otra parte, la importancia que le atribuyen los profesores de Lenguaje y Comunicación a la escritura supera la mera utilización de elementos lingüísticos para la comunicación, ya que esperan que los estudiantes sean capaces de desarrollar capacidades cognitivas de nivel superior.

Las teorías personales que expresan los profesores entrevistados sobre la escritura tienen como consecuencia decisiones sobre la frecuencia con que realizan actividades para promover su desarrollo, las metodologías implementadas, las actividades y la organización del aula. Sobre la frecuencia con que se realizan tareas de escritura en el aula, las opiniones de los docentes reflejan las limitaciones que se dan en el plano curricular e institucional (TSUI, 1996). Al parecer, la rigidez del sistema educativo tendría un impacto en las teorías personales de los profesores de Inglés, puesto que sus decisiones sobre qué enseñar estarían condicionadas por la institución y las políticas establecidas. En este contexto, se observa en el discurso del docente de Inglés que en su quehacer pedagógico influyen factores externos que condicionan los énfasis con que se enseñan las habilidades. Otra de las variables externas tiene que ver con el nivel de desarrollo de las competencias comunicativas 
del estudiante y con las facilidades que ofrece el establecimiento educativo para la enseñanza del inglés como lengua extranjera. Entre los factores internos que influyen en el profesor de inglés, se encuentra su experiencia como aprendiz. En esta línea nuestros hallazgos son concordantes con lo señalado por Burns (1992) cuando afirma que uno de los aspectos que determinantes en la enseñanza de la escritura es la naturaleza del lenguaje y su relación con el aprendizaje inicial que el docente experimentó durante su propia formación.

Cuando revisamos los análisis del discurso de los profesores de Lenguaje y Comunicación, encontramos que expresan su experiencia con la escritura como una actividad más positiva. Parece ser que la existencia de menos obstáculos durante su aprendizaje les permite desarrollar mejor y de manera más frecuente la tarea de escribir.

Sobre la implementación metodológica, advertimos que tanto los profesores de Lenguaje y Comunicación como los de Inglés utilizan la estrategia de modelamiento como una fase de la enseñanza de la escritura. Sin embargo, identificamos que el docente de Inglés presenta una aproximación predominantemente bottom-up para la enseñanza de la escritura, es decir, enseña a partir del dominio de unidades menores para elaborar el texto; mientras el docente de Lenguaje y Comunicación adopta una perspectiva top - down, esto es, parte desde la organización global hacia los aspectos de redacción local.

En términos de las estrategias utilizadas para la enseñanza de la escritura, los docentes coinciden con uno de los factores descritos por Burns (1992). La autora señala que puede influir en las teorías personales de los docentes la naturaleza del aprendizaje inicial de una lengua y las estrategias que resultaron relevantes durante su aprendizaje. En este contexto, el estudiante que aprende a escribir en una lengua extranjera se sitúa en una etapa de aprendizaje diferente a la de un estudiante que escribe en su lengua materna. Ello explicaría la diferente naturaleza de las decisiones metodológicas respecto de la enseñanza de la escritura.

Llama la atención la idea compartida entre los docentes de Inglés y de Lenguaje y Comunicación sobre el aprendizaje de la escritura como un proceso netamente individual. Esta teoría personal puede estar influenciada por la experiencia del profesor como aprendiz. Como lo explican Eisenstein - Ebsworth y Schweers (1997) y Woods (2011) la mayoría de los profesores han recibido una instrucción basada en un modelo conductista caracterizado por una enseñanza frontal y una disposición de aula en filas, lo que dificulta la interacción entre los alumnos y promueve el trabajo individual. En Chile, las clases no solo replican lo señalado por los autores, sino que la cantidad de estudiantes en las aulas de establecimientos 
públicos supera, en muchas ocasiones, a los 40 alumnos. Tal situación dificulta el desarrollo de un buen trabajo en grupo.

Al mismo tiempo, las opiniones de los docentes expresan que enseñar a escribir es una actividad compleja que requiere dedicación y tiempo. Básicamente, lograr que un estudiante escriba un buen texto en inglés o español requiere de varias revisiones del profesor. Como señala Caldera (2006),

es importante que el docente le explique y demuestre al estudiante que el primer intento de escritura en ningún caso puede ser considerado como la composición final, ya que a medida que el alumno-escritor regrese a lo ya escrito podrá verificar si está o no produciendo el efecto que realmente quiere (p. 87).

Por lo tanto, el análisis de los datos revela que las teorías personales, especialmente, de los docentes de Lenguaje y Comunicación están predominantemente asociadas al enfoque de proceso. Por su parte, el profesor de Inglés elabora su teoría personal sobre escritura como una habilidad que resulta muy difícil de desarrollar. Para Kessler (2009) y Warschauer (2010) el profesor expresa esta dificultad en su actuación pedagógica, puesto que se ve enfrentado a diferentes barreras que probablemente trata de evitar para no convertir su instrucción en algo tedioso y demasiado complicado para sus estudiantes.

Muchas de las teorías personales identificadas en esta investigación reflejan lo que a juicio de Eysenck (2001) y Feryok (2010) constituyen las teorías personales declarativas. Tales constructos son propios de aquellos docentes que estarían más enfocados en enseñar conceptos y estructuras que en desarrollar habilidades propiamente tales. Desde esta perspectiva, surge una inquietud en el sentido de si las teorías personales de los profesores entrevistados son resultado de sus propias convicciones pedagógicas o son el resultado del contexto laboral. En otras palabras, las teorías personales de los docentes se enfrentarían con la realidad compleja de las prácticas educativas y las tensiones que surgen entre las creencias propias y la realidad del aula.

\section{REFERÊNCIAS BIBLIOGRÁFICAS}

ALMARZA, G. (1996). Student foreign language teachers' growth. In D. Freeman \& J. C. Richards (eds.), TeacherLearning in Language Teaching (pp. 50-78). Cambridge: Cambridge University Press.

BAILEY K., CURTIS, A.; NUNAN, D. (2001). Pursuing professional development.Boston: NewbutyHouse. 
BISQUERRA, R. (2009). Metodología de la investigación educativa. $2^{\text {a }}$ edición. España: Editorial La Muralla, S.A.

BORG, S. (1999). Teachers' theories in grammar teaching. ELT Journal Volume 53/3July, pp.149-156 Oxford University Press.

BORG, S. (2003). Teacher cognition in language teaching: a review of research on what language teachers think, know, believe, and do. Language Teaching, 36(2), pp.81-109. Cambridge University Press.

BURNS, A. (1992). Teacher beliefs and their influence on classroom practice. Prospect, 7 (3), pp.56-66.

CABAROGLU, N.; ROBERTS, J. (2000). Development in student teachers' pre-existing beliefs during a 1-Year PGCE programme. System, 28 (3), pp.387-402.

CASTRO, P.; MÉNDEZ, M.; SERCU, L., (2003). La cultura en el aula de lenguas extranjeras: análisis de las percepciones de los profesores de secundaria respecto a los objetivos y las actividades de aprendizaje cultural. Disponible en http://sedll. org/es/admin/uploads/congresos/15/act/131/Castro_Prieto.pdf: Acceso el: 16 nov. 2012.

CALDERA, R. (2006). Enseñanza-aprendizaje de la escritura. Una propuesta a partir de la investigaciónacción. Mérida, Venezuela: Consejo de Publicaciones de la Universidad de Los Andes.

DIAZ, C.; SOLAR M. I. (2011). El lado oculto de la enseñanza: las creencias pedagógicas de docentes universitarios de Inglés acerca de su rol y el de sus estudiantes en el proceso didáctico.In: Arancibia, B., Díaz, C. y Tapia, M. (Eds) Enseñanza y Aprendizaje del Inglés como Lengua Extranjera. Concepción: Editorial Universidad Católica de la Santísima Concepción, pp. 107-142.

EYSENCK, M.W. (2001). Principles of Cognitive Psychology. Philadelphia, PA: Psychology Press, Ltd. Taylor and Francis Inc.

EISENSTEIN-EBSWORTH, M.; SCHWEERS C. W. (1997). What researchers say and practitioners do: Perspectives on conscious grammar instruction in the ESL classroom. Applied Language Learning, 8, pp.237-60.

FENSTERMACHER, G. D. (1994). The knower and the known: The nature of knowledge in research on teaching. Reviewof Research in Education, 20, pp.1-54.

FERYOK, A. (2010). Language teacher cognitions: Complex dynamic systems? System, 38 : 2, pp. 272-279.

FLESCH, R. (1955). WbyJobnny can't read. New York: Harper Row.

FLOWER, L.; HAYES, J. (1981). A Cognitive Process. Theory of Writing.Collage Composition and Communication, 32, pp. 365-387.

GATBONTON, E. (1999). Investigating experienced ESL teachers' pedagogical knowledge. The Modern LanguageJournal, 83 (1), pp.35-50.

GOLOMBEK, P. R. (1998). A study of language teachers'personal practical knowledge. TESOL Quarterly, 32 (3), pp.447-64.

GRAHAM, S.; HARRIS, K. R.; MACARTHUR, C.; FINK, B. (2002). Primary grade teachers' theoretical orientations concerning writing instruction: Construct validation and a nationwide survey. Contemporary EducationalPsychology, 27, pp.147-166.

HILLOCKS, G.JJr. (1984). What works in teaching composition: A meta-analysis of experimental treatment studies. AmericanJournal of Education, 93, pp.133-170. 
KESSLER, G. (2009). Student-initiated attention to form in wiki-based collaborative writing. Language Learning \& Technology, 13, (1), pp.79-95.

LIPSON, M.; MOSENTHAL, J.; DANIELS, P.; WOODSIDE-IRON, H. (2000). Process writing in the classrooms of eleven fifth-grade teachers with different orientations to teaching and learning. The Elementary School Journal, 101(2), pp.209-232.

MERTLER, C. (2006). Action Research. Teachers as Researchers in the Classroom. London: Sage Publications.

MILES, M.B.; HUBERMAN, A. (1994).Qualitative data analysis: an expanded sourcebook. Newbury Park CA: Sage.

MILLS, G.E. (2007). Action Research. A Guide for the Teacher Researcher. NewJersey:Pearson Education

NUNAN, D. (1992). The teacher as decision-maker. Perspectives on Second Language Teacher Education.Eds. J. Flowerdew, M. Brock \& S. Hsia: Hong Kong: City Polytechnic. pp. $135-65$.

ORTON, R. E. (1996). How can teacher beliefs about student learning be justified? Curriculum Inquiry, 26 (2), pp.133-46.

PAJARES, M. (1992). Teachers' beliefs and educational research: Cleaning up a messy construct. Review of EducationalResearch, 62, pp.303-332.

PENG. J. (2011). Changes in language learning beliefs during a transition to tertiary study: The mediation of classroom affordances. System, Volume 39, Issue 3, pp.314-324.

PHIPPS S, BORG, S. (2007). Exploring the relationship between teachers' beliefs and their classroom practice.The Teacher Trainer. 21.3, pp.17-19.

SENDAN, F.; ROBERTS, J. (1998). Orhan: a case study in the development of a student teacher's personal theories. Teachers and Thinking. Theory and Practice, 4, pp.229244.

SPADA, N.; MASSEY, M. (1992). The role of prior pedagogical knowledge in determining the practice of novice ESL teachers. Eds.J. Flowerdew, M. Brock \&S.Hsia, Perspectives on Second LanguageTeacher Education. Hong Kong: City Polytechnic. pp.23-37.

TSUI, A. B. M. (1996). Learning how to teach ESL writing. Eds. D. Freeman \& J. C. Richards, Teacher Learning inLanguage Teaching. Cambridge: Cambridge. University Press. pp.97-119

WARSCHAUER, M. (2010). Invited commentary: New tools for teaching writing. En Language Learning \& Technology, 14, (1), pp.3-8.

WOODS, D. (1996). Teacher Cognition in Language Teaching. Cambridge: Cambridge University Press.

WOODS, D. (2011). Two dimensions of teacher knowledge: The case of communicative language teaching. En System, 39(3), pp. 381-390.

Recebido: $12 / 12 / 2012$

Aceito: 22/04/2013 